Published in final edited form as:

Eur J Prev Cardiol. 2019 March ; 26(4): 433-436. doi:10.1177/2047487318800511.

\title{
Meta-analysis of long-term outcomes of percutaneous coronary intervention versus medical therapy in stable coronary artery disease
}

\author{
Safi U Khan ${ }^{1}$, Maninder Singh ${ }^{2}$, Ahmad N Lone ${ }^{1}$, Muhammad Shahzeb Khan ${ }^{3}$, Urooj \\ Fatima $^{4}$, Abu Bakar Saad ${ }^{5}$, Haris Riaz ${ }^{6}$, Isaac Opoku-Asare ${ }^{4}$, and Edo Kaluski ${ }^{2}$ \\ ${ }^{1}$ Department of Medicine, West Virginia University, USA \\ ${ }^{2}$ Department of Cardiovascular Medicine, Guthrie Health System/Robert Packer Hospital, USA \\ ${ }^{3}$ Department of Medicine, Cook County Hospital, USA \\ ${ }^{4}$ Department of Cardiovascular Medicine, Howard University Hospital, USA \\ ${ }^{5}$ Department of Cardiovascular Medicine, Nishtar Hospital, Pakistan \\ ${ }^{6}$ Department of Cardiovascular Medicine, Cleveland Clinic, USA
}

Percutaneous coronary intervention (PCI) improves survival and reduces the risk of recurrent myocardial ischemia in patients with acute coronary syndrome. ${ }^{1}$ Conversely, the role of PCI in patients with stable coronary artery disease (CAD) remains controversial. ${ }^{2}$ In the ORBITA trial, PCI was not associated with significant improvement in exercise time or angina frequency compared with a sham procedure, ${ }^{2}$ whereas long-term outcomes of the FAME 2 trial showed that a fractional flow reserve (FFR)-guided PCI strategy was superior to medical therapy (MT) in terms of cardiovascular outcomes. ${ }^{3}$ To address this controversy, we have performed an updated meta-analysis comparing the efficacy of PCI versus MT at a mean follow-up duration of 5 years.

A comprehensive search strategy was devised using MEDLINE, EMBASE and CENTRAL (inception to 30 May 2018) to identify randomized controlled trials (RCTs) with a sample size greater than 400 patients (to avoid small study effects) ${ }^{4}$ and over 4 years follow-up duration. We included trials reporting PCI with stent implantation in $70 \%$ or more of the patients and statin therapy in 50\% or more patients in the study population. The outcomes of interest were all-cause mortality, myocardial infarction (MI), stroke, cardiovascular mortality, revascularization and angina relief. Quality assessment of each trial was

\footnotetext{
Article reuse guidelines: sagepub.com/journals-permissions

Corresponding author: Safi U Khan, Department of Medicine, West Virginia University, Morgantown, WV 26505, USA., safinmc@gmail.com.

The first two authors are primary co-authors.

Author contribution

SUK contributed to the conception and design of the work. ANL, MSK and UF contributed to the data acquisition, analysis and interpretation of data for the work. SUK and MS drafted the manuscript. HR, IOA and EK critically revised the manuscript. All authors gave final approval and agree to be accountable for all aspects of the work ensuring integrity and accuracy.

Declaration of conflicting interests

The authors declared no potential conflicts of interest with respect to the research, authorship, and/or publication of this article.
} 
performed using the Cochrane risk of bias tool. The literature search, data extraction and bias risk assessment was done by authors ANL, MSK and UF independently. Estimates were pooled using the DerSimonian and Laird random effects model and reported as risk ratio (RR) with 95\% confidence interval (CI). Heterogeneity was quantified by $\boldsymbol{I}^{2}$ with values greater than $75 \%$ consistent with high grade heterogeneity. Publication bias was assessed using Egger's regression test. Analyses were conducted at 5\% significance. Comprehensive Meta-Analysis (version 3) was used for meta-analysis.

Five RCTs ( 8117 patients) $)^{3,5-8}$ were finalized in this meta-analysis (Table 1). At a mean follow-up duration of 5 years, there were no significant differences between PCI and MT in terms of all-cause mortality (RR $0.99,95 \%$ CI $0.86-1.15, P=95 \%, P=0 \%$ ), MI (RR 1.00 , $0.95 \%$ CI $0.80-1.25, P=0.99, I^{2}=54 \%$ ), stroke (RR $1.14,95 \%$ CI $0.83-1.57, P=0.43, P^{2}=$ $0 \%$ ), revascularization $0.61,95 \%$ CI $0.28-1.31, P=0.21, I^{2}=97 \%$ ), cardiovascular mortality (RR 1.06, 95\% CI 0.82-1.36, $P=0.67, I^{2}=0 \%$ ) or angina relief (RR $1.03,95 \%$ CI $0.93-$ $1.15, P=0.54, I^{2}=66 \%$ ) (Figure 1). Egger's regression test did not detect publication bias $(P($ two-tailed $)=0.19)$.

This meta-analysis suggests that in patients with stable CAD, PCI was not associated with a reduction in cardiovascular outcomes, angina relief or survival benefit compared with MT at 5 years mean follow-up duration. The former meta-analyses included studies with shorter follow-ups with the lesser use of stents, which is contrary to the current standard of care. ${ }^{9,10}$ The current study is unique because of the inclusion of more contemporary trials with longer follow-up durations and studies in which the use of stents ranged from $72 \%$ to $97 \%$ in the PCI arm. Therefore, these results should be considered more robust and reliable in view of current practice.

This study revalidates the beneficial role of optimal MT in patients with stable CAD. A recent meta-analysis of 10 studies (106,002 patients) confirmed that evidence-based medication regimens (beta-blockers, angiotensin-converting enzyme inhibitors, antiplatelet therapy and statins) were associated with a reduced risk of cardiovascular events and mortality. ${ }^{11}$ However, it is important to mention that intensive MT should be coupled with aggressive risk modification to generate favorable cardiovascular outcomes. For instance, in a post hoc analysis of the BARI-2D trial, current or former smoking was associated with greater than twice the risk of all-cause mortality in patient with diabetes and stable CAD receiving MT or PCI. ${ }^{12}$ Therefore, it is critical that physicians should enforce the importance of risk modification while prescribing guideline-directed MT.

This meta-analysis has limitations which are mainly related to the limitations inherent in the included RCTs. As drug-eluting stents were approved only after 2004, PCI was performed using bare-metal stents in the majority of the RCTs with the exception of the FAME 2 trial, ${ }^{3}$ in which second generation drug-eluting stents were used as part of the study protocol. In some trials, balloon angioplasty alone without stenting was performed in more than $25 \%$ of the patients. ${ }^{7}$ Also, there were a significant number of patients who crossed over from the MT to the PCI arm in these trials, which is not factored in in this meta-analysis. Another limitation is the heterogeneity in inclusion criteria and eligibility for PCI in these RCTs. In the OAT trial, patients with stable CAD were included who had persistent total occlusion of 
the infarct-related coronary artery 3-28 days after MI. ${ }^{7}$ Only the FAME 2 trial included an objective criterion, FFR for inclusion in the study. ${ }^{3}$ In other trials, FFR was not mandatory to assess the significance of the coronary lesion. The majority of the patients probably represented a low-risk cohort, as evident from an average left ventricular ejection fraction of over $45 \%$ in all these trials. Furthermore, only a small proportion of patients with multivessel disease were included in these trials. Only one third of patients in the COURAGE and BARI-2D trials had three-vessel disease, ${ }^{5,6}$ while the FAME 2 trial included only $9 \%$ of patients with three-vessel disease. ${ }^{3}$

In conclusion, in patients with stable CAD, PCI was not associated with a reduction in cardiovascular outcomes, angina relief or improved survival compared with MT at a mean follow-up duration of 5 years.

\section{Funding}

The authors received no financial support for the research, authorship, and/or publication of this article.

\section{References}

1. Windecker S, Kolh P, Alfonso F, et al. 2014 ESC/EACTS Guidelines on myocardial revascularization: the Task Force on Myocardial Revascularization of the European Society of Cardiology (ESC) and the European Association for Cardio-Thoracic Surgery (EACTS). Developed with the special contribution of the European Association of Percutaneous Cardiovascular Interventions (EAPCI). Eur Heart J 2014; 35: 2541-2619.

2. Al-Lamee R, Thompson D, Dehbi HM, et al. Percutaneous coronary intervention in stable angina (ORBITA): a double-blind, randomised controlled trial. Lancet (London, England) 2018; 391: 3140.

3. Xaplanteris P, Fournier S, Pijls NHJ, et al. Five-year outcomes with PCI guided by fractional flow reserve. N Engl J Med 2018; 379: 250-259. [PubMed: 29785878]

4. Schwarzer G, Carpenter JR and G. Small-study effects in meta-analysis In: Meta-Analysis with R. Cham: Springer International Publishing, 2015, pp. 107-141.

5. Boden WE, O'Rourke RA, Teo KK, et al. Optimal medical therapy with or without PCI for stable coronary disease. N Engl J Med 2007; 356: 1503-1516. [PubMed: 17387127]

6. Frye RL, August P, Brooks MM, et al. A randomized trial of therapies for type 2 diabetes and coronary artery disease. N Engl J Med 2009; 360: 2503-2515. [PubMed: 19502645]

7. Hochman JS, Lamas GA, Buller CE, et al. Coronary intervention for persistent occlusion after myocardial infarction. N Engl J Med 2006; 355: 2395-23407. [PubMed: 17105759]

8. Hueb W, Lopes NH, Gersh BJ, et al. Five-year follow-up of the Medicine, Angioplasty, or Surgery Study (MASS II): a randomized controlled clinical trial of 3 therapeutic strategies for multivessel coronary artery disease. Circulation 2007; 115: 1082-1089. [PubMed: 17339566]

9. Stergiopoulos K, Boden WE, Hartigan P, et al. Percutaneous coronary intervention outcomes in patients with stable obstructive coronary artery disease and myocardial ischemia: a collaborative meta-analysis of contemporary randomized clinical trials. JAMA Intern Med 2014; 174: 232-240. [PubMed: 24296791]

10. Thomas S, Gokhale R, Boden WE, et al. A meta-analysis of randomized controlled trials comparing percutaneous coronary intervention with medical therapy in stable angina pectoris. Can J Cardiol 2013; 29: 472-482. [PubMed: 23010084]

11. Du L, Cheng Z, Zhang Y, et al. The impact of medication adherence on clinical outcomes of coronary artery disease: a meta-analysis. EurJ Prev Cardiol 2017; 24: 962-970. [PubMed: 28436725] 
12. Khan AA, Chung MJ, Novak E, et al. The long-term risk of smoking in diabetic patients with stable ischemic heart disease treated with intensive medical therapy and lifestyle modification. EurJ Prev Cardiol 2017; 24: 1506-1514. [PubMed: 28517955] 


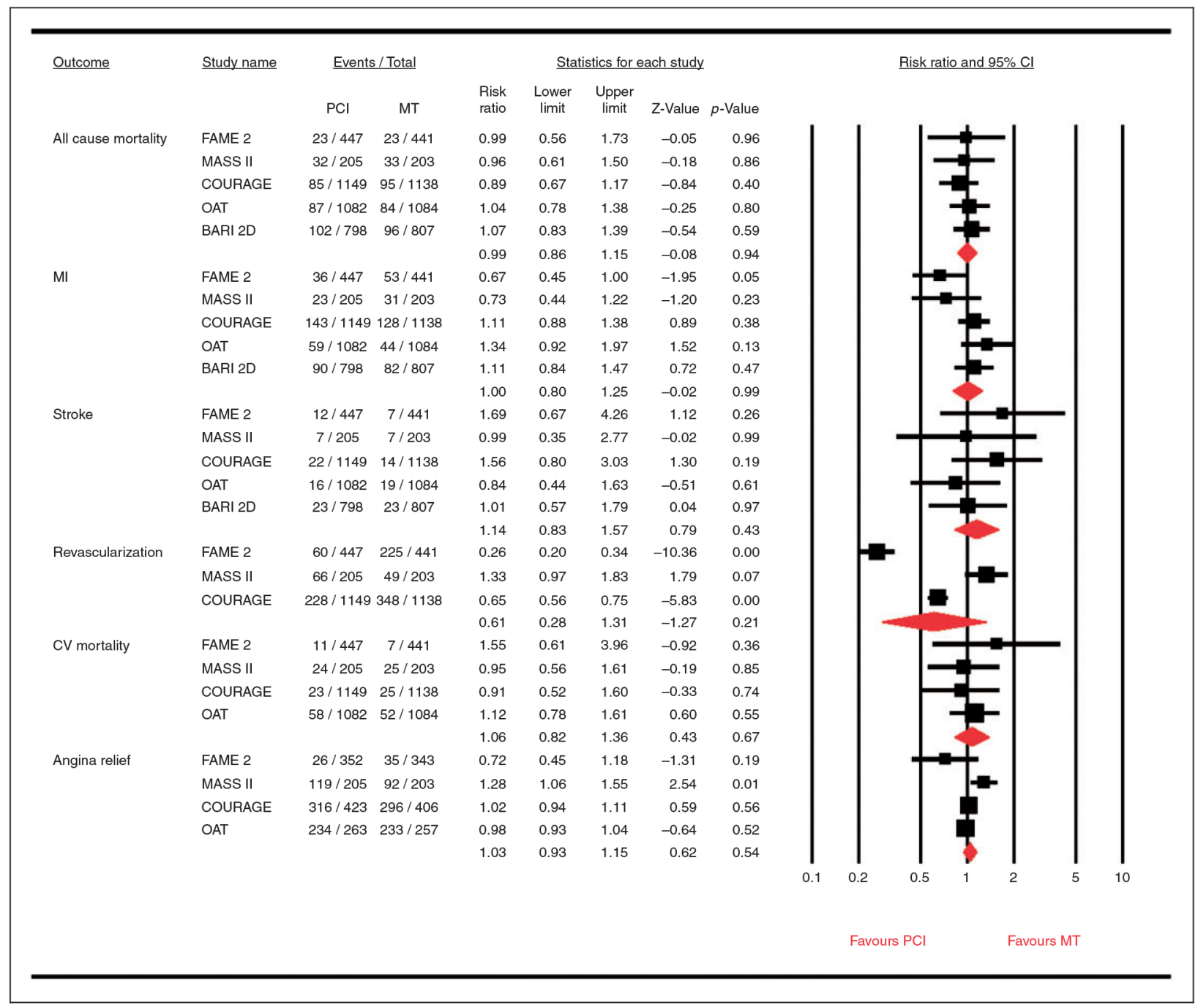

Figure I.

Forest plot comparing percutaneous coronary intervention (PCI) versus medical therapy (MT) in stable coronary artery disease patients.

MI: myocardial infarction; CV: cardiovascular; CI: confidence interval. 


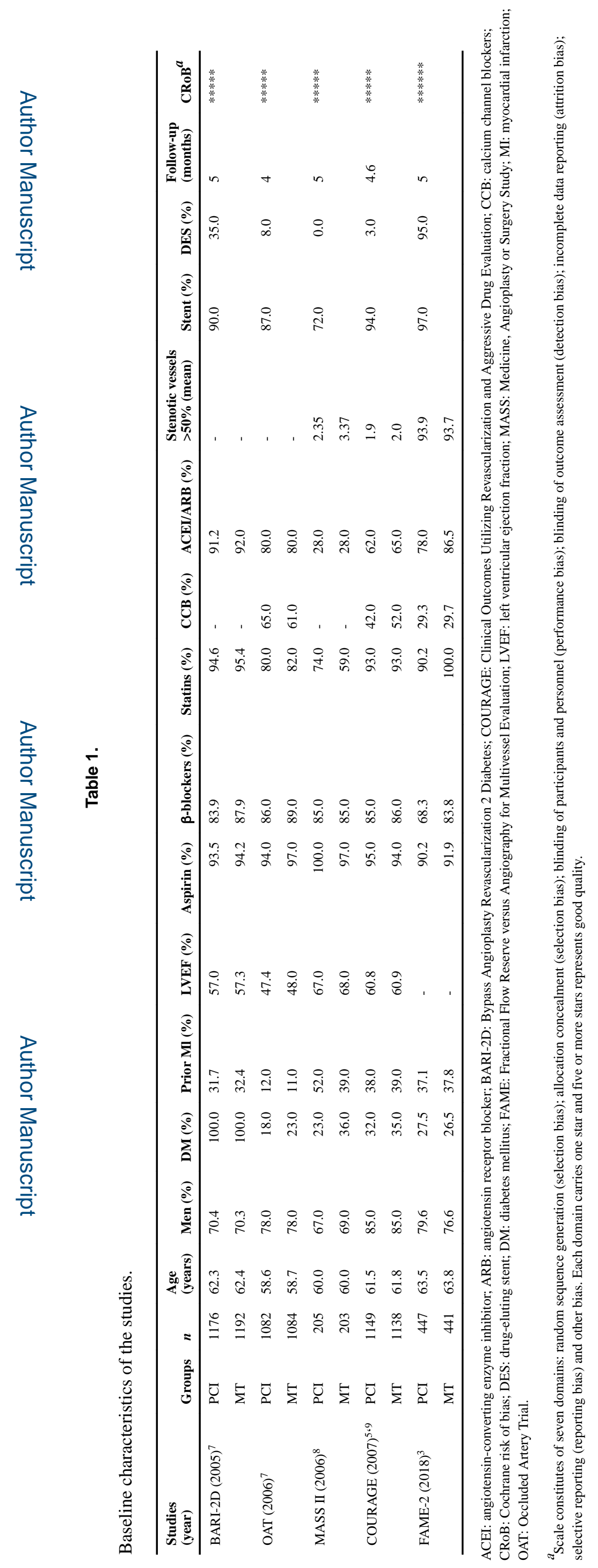

